Max-Planck-Institut für demografische Forschung

Max Planck Institute for Demographic Research

Konrad-Zuse-Strasse 1 - D-18057 Rostock - GERMANY

Tel +49 (0) 3812081 - 0; Fax +49 (0) 3812081 - 202;

http://www.demogr.mpg.de

MPIDR WORKING PAPER WP 2003-003

JANUARY 2003

\title{
Räumlicher Kontext und das \\ Heiratsverhalten westdeutscher Männer in den 1980er und 1990er Jahren
}

Karsten Hank (hank@ demogr.mpg.de)

This working paper has been approved for release by: Jan M. Hoem (hoem@demogr.mpg.de)

Head of the Laboratory of Contemporary European Fertility and Family Dynamics.

(C) Copyright is held by the authors.

Working papers of the Max Planck Institute for Demographic Research receive only limited review.

Views or opinions expressed in working papers are attributable to the authors and do not necessarily reflect those of the Institute. 


\title{
Räumlicher Kontext und das Heiratsverhalten westdeutscher Männer in den 1980er und 1990er Jahren
}

\author{
Karsten Hank ${ }^{\star}$
}

Zusammenfassung: In diesem Beitrag wird der Zusammenhang zwischen Merkmalen des räumlichen Kontexts und dem Übergang zur ersten Ehe bei westdeutschen Männern in den 1980er und 1990er Jahren untersucht. Hierzu werden im empirischen Teil unter Verwendung von Individualdaten des Sozio-oekonomischen Panels (SOEP) und Informationen über Stadt- und Landkreise zeitdiskrete logistische Mehrebenenmodelle geschätzt. Die Ergebnisse der multivariaten Analyse bestätigen die herausragenden Bedeutung der wirtschaftlichen Stellung eines Mannes für seine Heiratsentscheidung. Darüber hinaus wird ein negativer Zusammenhang zwischen der aggregierten Erwerbsbeteiligung von Frauen auf dem regionalen Arbeitsmarkt und der Übergangswahrscheinlichkeit zur ersten Ehe bei Männern festgestellt. Dies könnte als Beleg für die in der Heiratsforschung verbreitete 'wirtschaftliche Unabhängigkeitshypothese' verstanden werden. Komplementär hierzu wird eine Interpretation der Frauenerwerbsquote als Indikator für regional unterschiedliche Ausprägungen von Geschlechterrollen und individueller Autonomie vorgeschlagen. Berücksichtigt man zusätzlich den in den hier geschätzten Modellen statistisch signifikanten latenten Kontexteffekt, ergeben sich deutliche Indizien dafür, dass die Heiratsneigung eines Mannes durch regionale sozio-kulturelle Milieus beeinflußt wird.

` Beitrag für den Workshop "Das 'vernachlässigte' Geschlecht in der Familienforschung: Analysen zum Heirats- und Geburtenverhalten von Männern in Deutschland" am 27./28. Februar 2003 in Rostock. Eine englische Fassung erschien 2002 unter dem Titel "The Geographic Context of Male Nuptiality in Western Germany During the 1980s and 1990s" in Demographic Research, 7 (15), S. 523-536, online: www.demographic-research.org.

Korrespondenzanschrift (ab 15. Februar 2003): Dr. Karsten Hank, Mannheimer Forschungsinstitut Ökonomie und demographischer Wandel ( MEA ); Universität Mannheim; L 13, 17; 68131 Mannheim. Email: hank@mea.uni-mannheim.de. 


\section{Einleitung}

Vor inzwischen zehn Jahren stellte Hans Bertram in einem Interview fest, „daß nur durch die Berücksichtigung kultureller und sozialräumlicher Faktoren ein angemessenes Bild der Lebenssituation von [...] Familien zu entwickeln ist.“ (Bertram 1993: 37) Seither sind eine Reihe zum Teil sehr elaborierter, jedoch weitgehend deskriptiver Studien regionaler Familien- und Haushaltsstrukturen in Deutschland entstanden (z.B. Bertram et al. 2000; Nauck und Bertram 1995). Es ist jedoch von Nauck (1995: 119) zurecht darauf hingewiesen worden, dass „,[e]ine wie immer verfeinerte Regionaltypologie [...] lediglich ein erster Schritt für das weitergehende Ziel sein [kann], die Basis für empirische Mehrebenen-Analysen zu legen, bei der Aggregat- und Individual-Daten für eine kontextualisierte Erklärung familiären Verhaltens miteinander verknüpft werden.“1

Entsprechende Analysen des Einflusses regionaler sozialer Kontexte auf die Familiengründung westdeutscher Frauen in den 1980er und 1990er Jahren unterstreichen die Notwendigkeit und Fruchtbarkeit eines solchen Ansatzes (Hank 2002; 2003). Die beiden wichtigsten Befunde dieser Untersuchungen auf Basis des Sozio-oekonomischen Panels sind erstens, dass regionale Unterschiede im Geburtenverhalten weitestgehend durch Kontrolle individueller Merkmale - vor allem Familienstand und Alter der Frau - erklärt werden können (vgl. auch Kopp 2000), und zweitens, dass es eine signifikante regionale Varianz im Heiratsverhalten gibt, die weder auf Bevölkerungskompositionseffekte noch auf strukturelle Kontexteffekte zurück-

\footnotetext{
${ }^{1}$ Vgl. Teachman und Crowder (2002) für einen Überblick über grundlegende methodische und konzeptuelle Aspekte im Zusammenhang mit der Verwendung von Mehrebenenmodellen in der Familienforschung.
} 
zuführen ist. Aus diesen Ergebnissen wird auf die Existenz raumgebundener soziokultureller Milieus geschlossen, die sich z.B. in der Akzeptanz nicht-ehelicher Lebensgemeinschaften oder in kollektiven Erwartungen hinsichtlich des Heiratsalters voneinander unterscheiden (vgl. Hank 2003).

Hieran anschließend untersucht die vorliegende Studie den Zusammenhang zwischen Merkmalen des räumlichen Kontexts, der auf der Ebene von Stadt- und Landkreisen definiert wird, und dem Übergang zur ersten Ehe bei westdeutschen Männern in den 1980er und 1990er Jahren. Abbildung 1 zeigt die räumliche Verteilung der rohen Heiratsziffern in den westdeutschen Kreisen für den entsprechenden Zeitraum. ${ }^{2}$ Ziel der Untersuchung ist es, herauszufinden, ob sich die Befunde der oben zitierten Analyse des Heiratsverhaltens von Frauen für Männer replizieren lassen, oder ob geschlechtsspezifische Kontexteinflüsse auf die individuelle Entscheidung zur Eheschließung festgestellt werden können (vgl. Lloyd und South 1996). Dies ist insofern bedeutsam, als die Rolle des Mannes als eigenständiges Subjekt im Prozeß der Familiengründung bislang von der einschlägigen Forschung weitgehend vernachlässigt wurde (z.B. Goldscheider und Kaufman 1996; Greene und Biddlecom 2001). ${ }^{3}$

2 Leider erlauben es die hier zur Verfügung stehenden Daten nicht, alters- oder geschlechtsspezifische Heiratsraten auszuweisen.

${ }^{3}$ In einem Überblicksartikel zur Fertilität von Männern in Industrieländern notiert Coleman (2000: 32), dass “[t]he dominant linkage of men's economic position in the marriage and fertility system of historical Western Europe with late and avoidable marriage led to the apparent paradox that while almost all analysis of the pattern and trend of fertility was conducted with respect to the employment and wage earning of men, the indices of fertility and reproduction universally employed related to women only and were based on women's rates. [...] Since the Second World War, and the overturning of this Malthusian apple-cart based on single (male) earner households, the balance of interest has moved further against men." 
[Abbildung 1]

\section{Heiratsentscheidungen aus regionaler Perspektive}

Wenn - wie in der westdeutschen Gesellschaft - Elternschaft üblicherweise in der Ehe stattfindet (z.B. Billari und Kohler 2002), gleichzeitig jedoch nicht-eheliche Partnerschaften eine sozial akzeptierte und kaum mehr benachteiligte Form des Zusammenlebens darstellen, gewinnt die Frage an Bedeutung, unter welchen Bedingungen Individuen heiraten (vgl. Waite et al. 2000).

Auf der individuellen Entscheidungsebene ist häufig argumentiert worden, dass die Heiratswahrscheinlichkeit insbesondere von Bildungsstand und sozioökonomischem Status, sowie vom familiären Hintergrund und Wertorientierungen abhängt (z.B. Clarkberg et al. 1995; Cooney und Hogan 1991; Oppenheimer 2000). Für Westdeutschland konnte Huinink (1995: 255 ff.) u.a. nachweisen, dass Männer aus Herkunftsfamilien mit höherem sozialen Status später heiraten. Gleiches gilt für jene, die sich in Ausbildung befinden, während erwerbstätige Männer - insbesondere solche, die in statushohen Berufen arbeiten - die höchsten Übergangsraten zur ersten Ehe aufweisen.

Mit Blick auf die kontextuellen Determinanten der Eheschließung hat sich die bisherige Forschung hauptsächlich mit der Rolle von Heiratsmärkten befaßt (z.B. Lichter et al. 1991), wo die Auswahl potenzieller Partnerinnen häufig in räumlich begrenzten sozialen Netzwerken erfolgt (South and Crowder 2000: 1069; Lengerer 2001). Bei der Analyse lokaler Heiratsmärkte ist es sinnvoll, zwischen der 'Quantität' und der 'Qualität' des Pools möglicher Partnerinnen zu unterscheiden. Die Ergebnisse einer Studie von Lloyd und South (1996) zeigen, dass ein Überschuß an Frauen den 
Eintritt von Männern in den Ehestand fördert, was im Einklang mit Theorien zum Suchverhalten auf Heiratsmärkten steht (z.B. Oppenheimer 1988). Bei zusätzlicher Berücksichtigung der Rolle von Qualitätserwägungen für Heiratsentscheidungen wird erwartet, dass die individuelle Neigung zu heiraten mit dem regionalen Angebot an wirtschaftlich attraktiven Partnerinnen steigt, da sich mit diesen die eigenen Ressourcen in vorteilhafter Weise poolen lassen (z.B. Lichter et al. 1991). Eine höhere Bildungsund Erwerbsbeteiligung von Frauen führt jedoch gleichzeitig zu einer größeren wirtschaftlichen Unabhängigkeit potenzieller Partnerinnen, was häufig als Ursache für eine Verzögerung von Eheschließungen betrachtet wird (vgl. hierzu auch die ausführliche Diskussion bei Oppenheimer [1997]).

Heiratsmärkte bilden jedoch nur einen Teil der weiter $\mathrm{zu}$ fassenden regionalen Opportunitätsstruktur (vgl. Hank 2002: Abschnitt 2.2). Unabhängig von der eigenen Einkommens- oder Erwerbssituation kann beispielsweise auch die individuelle Wahrnehmung der allgemeinen wirtschaftlichen Lage die jeweilige Bereitschaft zur Familiengründung beeinflussen (z.B. Huinink 1995: 251). Schlechte Arbeitsmarktperspektiven könnten den Eintritt in eine Ehe verzögern, da unsicher ist, inwieweit der Mann (gegenwärtig oder zukünftig) seiner traditionellen Rolle als Haupternährer der Familie nachkommen kann.

Der wahrscheinlich meistgenannte kontextuelle Einflußfaktor auf individuelles Verhalten ist der Urbanisierungsgrad einer Region (z.B. Huinink und Wagner 1989; Linde 1988: 183 ff.). Dabei wird angenommen, dass die räumlichen Kategorien 'Stadt' und 'Land' durch unterschiedliche sozio-kulturelle Milieus charakterisiert werden können, insbesondere mit Blick auf die in der jeweiligen Bevölkerung dominierenden familienbezogenen Einstellungen und Werte. Außerdem ist anzunehmen, dass soziale 
Kontrolle mit zunehmender Bevölkerungsgröße abnimmt, da ländliche Regionen im Allgemeinen durch eine stärkere Einbindung von Individuen in die örtliche Gemeinschaft gekennzeichnet sind (vgl. Linde 1988: 184 f.; South und Crowder 2000: 1073).

Da sich die Fragestellung dieser Untersuchung nicht auf die Partnerschaftsgründung an sich, d.h. auf die Wahl einer bestimmten Partnerin, bezieht, sondern auf den Übergang zur ersten Ehe, d.h. auf die Wahl einer bestimmten Partnerschaftsform, könnten kollektive Verhaltenserwartungen der sozialen Umwelt möglicherweise von größerer Bedeutung für die individuelle Entscheidungsfindung sein, als strukturelle Merkmale des Heiratsmarktes. Regionale Unterschiede im Modernisierungs- und Individualisierungsgrad sind in diesem Zusammenhang häufig genannte mögliche Determinanten der räumlichen Verbreitung verschiedener Formen partnerschaftlichen Zusammenlebens (z.B. Klein 2000: 61f.; Lichter et al. 1991: 847). Obwohl man annehmen kann, dass sich normative Erwartungen bezüglich des Alters und der Reihenfolge, in denen bestimmte familiäre Ereignisse stattfinden sollten, je nach (sozialräumlichem) Kontext unterscheiden, sind sie empirisch nur schwer zu messen (z.B. Marini 1984). Die Berücksichtigung unbeobachteter regionaler Heterogenität in der multivariaten Analyse gewinnt hierdurch zusätzlich an Bedeutung.

\section{Daten und Methode}

\subsection{Datenbasis und Variablen}

Datenbasis für diese Studie ist das Sozio-oekonomische Panel des DIW Berlin (vgl. SOEP-Gruppe 2001). Dabei handelt es sich um eine in Westdeutschland seit 1984 jährlich durchgeführte Längsschnittuntersuchung privater Haushalte. Die vollständige 
Heiratsbiographie aller an der Befragung teilnehmenden Personen ist im Datensatz enthalten, wobei sich die vorliegende Analyse allein auf die erste Eheschließung in Westdeutschland lebender Männer bis zum Jahr 1999 bezieht.

Den Individualdaten des SOEP können Informationen über die Landkreise und kreisfreien Städte, in denen die Befragten leben, zugespielt werden. Die DJI Regionaldatenbank enthält Kreisindikatoren für die zweite Hälfte der 1980er Jahre, während entsprechende Informationen für die Mitte der 1990er Jahre in der Statistik regional Datenbank der Statistischen Ämter des Bundes und der Länder zu finden sind. Da die meisten interessierenden Regionalvariablen nur für jeweils einen Zeitpunkt in den 1980er und 1990er Jahren vorliegen, können zeitabhängige Kontextmerkmale ihre Ausprägung nicht jährlich ändern, sondern lediglich zwischen zwei als gleich lang definierten Zeiträumen, deren erster die Jahre 1984 bis 1991 umfaßt, während der zweite die Periode 1992 bis 1999 abdeckt.

Untersucht werden westdeutsche und ausländische Männer im Alter von 20 bis 40 Jahren. Personen, die während des Untersuchungszeitraums innerhalb Westdeutschlands umziehen, werden an ihren neuen Wohnort verfolgt. Damit besteht die hier verwendete Stichprobe aus 2.280 unverheirateten Männern in 300 Stadt- und Landkreisen. Da jedes Individuum mehrfach beobachtet werden kann, gehen insgesamt 15.223 Beobachtungen in die Analyse ein. Die Anzahl der Ereignisse (hier: Erstheiraten) im Untersuchungszeitraum beträgt 850 . Weitere deskriptive Statistiken finden sich in Tabelle 1.

Folgende individuelle (d.h. personenbezogene) Kontrollvariablen werden in die Regression aufgenommen: 
- das Alter und - da eine nicht-lineare Beziehung zur abhängigen Variablen angenommen wird - das quadrierte Alter der Befragten,

- zeitabhängige binäre Variablen, die den höchsten (beruflichen) Bildungsabschluss zum Zeitpunkt des jeweiligen Interviews angeben: in Ausbildung, kein Ausbildungsabschluss, beruflicher Ausbildungsabschluss (Referenzkategorie), Universitätsabschluss,

- eine zeitabhängige binäre Variable, die den Wert 1 annimmt, wenn der Befragte vollzeit erwerbstätig ist,

- sowie eine zeitunabhängige binäre Variable, die den Wert 1 annimmt, wenn der Befragte Ausländer ${ }^{4}$ ist.

Das Hauptaugenmerk gilt jedoch den Kontextmerkmalen auf der Kreisebene:

- der durchschnittliche Frauenanteil an der Bevölkerung im Alter von 20 bis 40 Jahren in der Periode 1995-1997 (in Prozent) (zeitunabhängig),

- die regionale Frauenerwerbsquote (in Prozent) (zeitabhängig, 1987/1995),

- eine zeitunabhängige binäre Variable, die den Wert 1 annimmt, wenn ein Kreis eine Bevölkerungsdichte von weniger als 150 Einwohnern pro $\mathrm{km}^{2}$ aufweist (= ländlicher Raum),

- und die regionale Arbeitslosenquote (in Prozent) (zeitabhängig, 1987/1996).

[Tabelle 1]

\footnotetext{
${ }^{4}$ Hierbei handelt es sich um Befragte der SOEP-Stichprobe B, also Griechen, Italiener, Spanier, Türken und Männer aus Ex-Jugoslawien, die bereits 1984 in der Bundesrepublik gelebt haben.
} 


\subsection{Das zeitdiskrete logistische Mehrebenenmodell}

Die Wahrscheinlichkeit, innerhalb eines Intervalls von einem Jahr zu heiraten, wird in dieser Untersuchung mit Hilfe zeitdiskreter logistischer Mehrebenenmodelle geschätzt (z.B. Barber et al. 2000). Jede Periode, in der ein Individuum beobachtet wird, stellt dabei eine unabhängige Beobachtung dar. Weil die erste Eheschließung ein einmaliges Ereignis ist, wird das Individuum nach Eintritt des Ereignisses von weiteren Beobachtungen ausgeschlossen. Die hieraus resultierende unzureichende Variation der (binären) abhängigen Variablen verhindert die Identifizierung unbeobachteter Individualeffekte, wie dies z.B. in einem herkömmlichen Paneldatenmodell möglich und notwendig wäre. Die 'Klumpung' mehrerer Individuen in einem gemeinsamen Kontext verletzt jedoch die Standardannahme unabhängiger Fehlerterme, was dazu führen kann, dass für die Aggregatvariablen ineffiziente Parameter und verzerrte Standardfehler geschätzt werden. Logistische Mehrebenenmodelle berücksichtigen diese Problematik.

In dieser Analyse wird angenommen, dass der Effekt der erklärenden Variablen in allen Stadt- und Landkreisen gleich ist. Die Regressionskonstante kann jedoch mit dem jeweiligen Kontext variieren, d.h. sie besteht aus einer fixen und einer zufälligen Komponente. Dieses random intercept Modell (vgl. Snijders und Bosker 1999: Kapitel 4) hat im konkreten Fall folgende Form:

$$
\log \left[p_{i j t} /\left(1-p_{i j t}\right)\right]=b_{0}+b_{1} x_{i j}+b_{2} z_{i j t}+b_{3} v_{j}+b_{4} w_{j t}+u_{0 j}
$$

wobei $p_{i j t}$ die Wahrscheinlichkeit angibt, mit der Individuum $i$ in Region $j$ im Jahr $t$ erstmals heiratet. Neben zeitabhängigen und zeitunabhängigen Individual- und Kontextvariablen findet sich auf der rechten Seite der Gleichung auch ein normalverteilter regionaler Zufallskoeffizient $u_{0 j}$. Er beschreibt die Abweichung jedes 
einzelnen Kontexts von $b_{0}$, d.h. vom fixen Teil der Regressionskonstante. Damit wird nicht nur für die Korrelation zwischen Individuen innerhalb desselben Kontexts kontrolliert, sondern es werden auch mögliche unbeobachtete regionale Effekte erfasst. Unterscheidet sich die Varianz von $u_{0 j}$ statistisch signifikant von 0 , liegt ein solcher latenter Kontexteffekt vor.

\section{Ergebnisse der multivariaten Analyse}

Um den Übergang in die erste Ehe zu analysieren werden zwei Modelle geschätzt. Während in Modell 1 nur für individuelle Merkmale der Männer kontrolliert wird, werden in Modell 2 zusätzlich die oben genannten Kontextvariablen aufgenommen. Beide Modelle berücksichtigen unbeobachtete Heterogeniät auf der Regionalebene. Die Regressionsergebnisse sind in Tabelle 2 dargestellt.

Hinsichtlich Vorzeichen und statistischer Signifikanz ergeben sich für die Koeffizienten der individuellen Kontrollvariablen die erwarteten Resultate (Modell 1). Der Alterseffekt wirkt nicht-monoton, d.h. die Heiratsneigung eines Mannes steigt zunächst mit zunehmendem Alter, sinkt jedoch wieder in späteren Lebensphasen. Bei den Ausbildungs- und Erwerbsvariablen zeigt sich, dass Männer in Ausbildung ebenso wie jene ohne Ausbildungsabschluss eine geringere Ehewahrscheinlichkeit aufweisen, während solche, die über einen Universitätsabschluss verfügen oder vollzeit erwerbstätig sind, ein höheres Heiratsrisiko haben, als Männer in der jeweiligen Referenzkategorie. Außerdem ist die Wahrscheinlichkeit eine Ehe einzugehen bei Ausländern höher als bei westdeutschen Männern.

In Modell 2 bleiben die Koeffizienten der Individualvariablen nach Aufnahme der Kontextindikatoren unverändert. Ebensowenig verringert sich der in beiden Modellen 
hochsignifikante regionale Zufallseffekt $\left(\sigma_{u}\right)$. Die Regionalvariablen tragen damit weder zur Erklärung der Varianz zwischen den Kreisen bei, noch verbessern sie in ihrer Summe die Güte des Modells. Dennoch lässt sich ein statistisch signifikanter Einfluß der Frauenerwerbsqoute feststellen: eine höhere Erwerbsbeteiligung von Frauen auf dem regionalen Arbeitsmarkt vermindert das 'Risiko' eines Mannes, im Beobachtungszeitraum eine erste Ehe einzugehen. $\mathrm{Zu}$ einem ähnlichen Ergebnis kommen auch Lloyd und South (1996: 1114) bei einer Analyse US-amerikanischer Daten.

[Tabelle 2]

\section{Diskussion}

Im vorliegenden Beitrag wird aus einer Mehrebenenperspektive heraus das Heiratsverhalten westdeutscher Männer untersucht. Die multivariate Analyse bestätigt zunächst den aus früheren Studien bereits bekannten starken Zusammenhang zwischen der wirtschaftlichen Stellung eines Mannes und der Heiratsentscheidung (z.B. Oppenheimer 2000). Gut ausgebildete und vollerwerbstätige Männer weisen demnach die höchste Wahrscheinlichkeit einer Eheschließung auf. Lebt ein Mann jedoch in einem Stadt- oder Landkreis mit einer hohen Frauenerwerbsquote, sinkt das Heiratsrisiko.

Diese negative Beziehung zwischen der Erwerbsbeteiligung von Frauen auf dem regionalen Arbeitsmarkt und dem Übergang zur ersten Ehe bei Männern könnte als Bestätigung von Hypothesen gewertet werden, die in der ökonomischen Unabhängigkeit von Frauen auf dem lokalen Heiratsmarkt eine der Hauptursachen für den Aufschub, bzw. das Ausbleiben von Eheschließungen sehen. Dieser zunächst 
plausibel erscheinende Erklärungsansatz erweist sich jedoch bei genauerer Betrachtung als nicht unproblematisch. In einem kritischen Literaturüberblick weist z.B. Oppenheimer (1997: 449) darauf hin, dass die sogenannte „Unabhängigkeithypothese“ nur durch solche Studien empirisch gestützt wird, die auf Aggregatdaten basieren. Untersuchungen auf der Grundlage von Individualdaten zeigen hingegen im Allgemeinen, dass eine höhere Bildung, Erwerbstätigkeit und das Einkommen von Frauen keinen, oder sogar einen tendenziell positiven Effekt auf die Heiratsneigung haben. In Übereinstimmung hiermit findet Hank (2003), dass Frauen ohne Ausbildungsabschluß niedrigere Übergangsraten zur ersten Ehe aufweisen als jene mit Berufsausbildung; ein Einfluß der Frauenerwerbsquote kann nicht nachgewiesen werden.

Es erscheint daher als sinnvoll, eine komplementäre Interpretation der Beziehung zwischen (aggregierter) Frauenerwerbstätigkeit und (individuellem) Heiratsverhalten von Männern in Betracht zu ziehen. Eine hohe Erwerbsbeteiligung von Frauen könnte z.B. nicht nur auf einen gesellschaftlichen Zusammenhang hinweisen, in dem diese in geringerem Maße ökonomisch von Männern abhängig sind, sondern sie könnte ebenso einen sozialräumlichen Kontext markieren, in dem gleichberechtigte Geschlechterrollen sowie die individuelle Autonomie (auch der Männer!) gegenüber jeglicher Art äußerer institutioneller Autorität verhältnismäßig stark ausgeprägt sind (vgl. Lesthaeghe und Neels 2002; Sackmann und Häussermann 1994). ${ }^{5}$ Folgt man diesem Ansatz, wären

\footnotetext{
${ }^{5}$ Differenziertere Indikatoren zur Beschäftigung von Frauen auf dem regionalen Arbeitsmarkt wie altersspezifische Erwerbsquoten, das Verhältnis von Teilzeit- und Vollzeitbeschäftigung, der Anteil erwerbstätiger verheirateter Frauen, oder die geschlechtsspezifische sektorale Differenzierung der Erwerbsbeteiligung - sind in den hier verwendeten Datenbanken leider nicht enthalten.
} 
regional unterschiedliche Normen, Einstellungen und Wertorientierungen eine wesentliche Ursache der zwischen den westdeutschen Stadt- und Landkreisen beobachteten Heterogenität im Heiratsverhalten. Wie bereits in Hanks (2003) Untersuchung der Familiengründung von Frauen, zeigt sich auch bei Männern ein statistisch signifikanter latenter Kontexteffekt auf das Risiko einer Eheschließung. Diese Befunde deuten darauf hin, dass sich partnerschafts- und familienbezogene kollektive Verhaltenserwartungen und Wertorientierungen in raumgebundenen soziokulturellen Milieus manifestieren können.

Dieser Erklärungsansatz ist jedoch keineswegs exklusiv, d.h. es existieren sehr wahrscheinlich noch weitere, hier nicht beobachtete und auch durch den regionalen Zufallskoeffizienten nur unzureichend erfasste Faktoren, die für das beobachtete geographische Heiratsmuster mitverantwortlich sein könnten. Daher bedarf es weiterer Forschungsanstrengungen, um genauer identifizieren zu können, welches die für das individuelle Handeln relevanten Kontextmerkmale sind, die schließlich zu regionalen Unterschieden im Heiratsverhalten von Männern und Frauen führen.

\section{Danksagung}

Diese Untersuchung ist vom Verfasser im Rahmen seiner Tätigkeit als wissenschaftlicher Mitarbeiter am Max-Planck-Institut für demografische Forschung in Rostock durchgeführt worden. Für kritische Anmerkungen danke ich Angelika Tölke und Gerda Neyer. Die multivariaten Modelle wurden während eines Gastaufenthaltes bei der SOEP-Gruppe am DIW Berlin geschätzt. 


\section{Literatur}

Barber, J.S., S.A. Murphy, W.G. Axinn \& J. Marples. 2000. Discrete-time multilevel hazard analysis. In: M.E. Sobel \& M.P. Becker (Hrsg.), Sociological Methodology, Vol.30. Washington: Blackwell Publishers, 201-235.

Bertram, H. 1993. Was bietet der Regionalansatz für Wissenschaft, Politik und Praxis? Diskurs, 1/93, 37-42.

Bertram, H., B. Nauck \& T. Klein (Hrsg.). 2000. Solidarität, Lebensformen und regionale Entwicklung. Opladen: Leske + Budrich.

Billari, F. \& H.-P. Kohler. 2002. The impact of union formation dynamics on first births in West Germany and Italy: Are there signs of convergence?" In: E. Klijzing \& M. Corijn (Hrsg.), Fertility and Partnership in Europe: Findings and Lessons from Comparative Research (Bd. 2). Genf \& New York: United Nations, im Druck.

Clarkberg, M., R.M. Stolzenberg \& L.J. Waite. 1995. Attitudes, Values, and Entrance into Cohabitational versus Marital Unions. Social Forces, 74 (2), 609-634.

Coleman, D.A. 2000. Male Fertility Trends in Industrial Countries: Theories in Search for Some Evidence. In: C. Bledsoe et al. (Hrsg.), Fertility and the Male Life-Cycle in the Era of Fertility Decline. Oxford: Oxford University Press, 29-60.

Cooney, T.M. \& D.P. Hogan. 1991. Marriage in an Institutionalized Life Course: First Marriage among American Men in the Twentieth Century. Journal of Marriage and the Family, 53 (1), 178-190.

Goldscheider, F.K. \& G. Kaufman. 1996. Fertility and Commitment: Bringing Men Back In. Population and Development Review, 22 (Suppl.), 87-99.

Greene, M.E. \& Biddlecom, A.E. 2001. Absent and Problematic Men: Demographic Accounts of Male Reproductive Roles. Population and Development Review, 26 (1), 81-115.

Hank, K. 2002. Regional Social Contexts and Individual Fertility Decisions: A Multilevel Analysis of First and Second Births in Western Germany. European Journal of Population, 18 (3), 281-299.

Hank, K. 2003. Eine Mehrebenenanalyse der Familiengründung westdeutscher Frauen in den Jahren 1984 bis 1999. Kölner Zeitschrift für Soziologie und Sozialpsychologie, 55 (1), im Druck. 
Huinink, J. 1995. Education, Work, and Family Patterns of Men: The Case of West Germany. In: H.-P. Blossfeld (Hrsg.), The new role of women: Family formation in modern societies. Boulder: Westview Press, 247-262.

Huinink, J. \& M. Wagner. 1989. Regionale Lebensbedingungen, Migration und Familienbildung. Kölner Zeitschrift für Soziologie und Sozialpsychologie, 41 (4), 669-689.

Klein, T. 2000. Partnerwahl und Partnerschaftsformen im regionalen Kontext. In: H. Bertram et al. (Hrsg.), Solidarität, Lebensformen und regionale Entwicklung. Opladen: Leske + Budrich, 59-82.

Kopp, J. 2000. Geburtenentwicklung in Ost- und Westdeutschland. In: H. Bertram et al. (Hrsg.), Solidarität, Lebensformen und regionale Entwicklung. Opladen: Leske + Budrich, 83-135.

Lengerer, A. 2001. Wo die Liebe hinfällt - ein Beitrag zur 'Geographie' der Partnerwahl. In: T. Klein (Hrsg.), Partnerwahl und Heiratsmuster. Sozialstrukturelle Voraussetzungen der Liebe. Opladen: Leske + Budrich, 133162.

Lesthaeghe, R. \& K. Neels. 2002. From the First to the Second Demographic Transition: An Interpretation of the Spatial Continuity of Demographic Innovation in France, Belgium and Switzerland. European Journal of Population, 18 (4), 325-360.

Lichter, D.T., F.B. LeClere \& D.K. McLaughlin. 1991. Local Marriage Markets and the Marital Behavior of Black and White Women. American Journal of Sociology, 96 (4), 843-867.

Linde, H. 1988: Das sozialökologische Gefälle der ehelichen Fruchtbarkeit im Prozeß der Nachwuchsbeschränkung in Deutschland. In: C. Höhn et al. (Hrsg.), Demographie in der Bundesrepublik Deutschland: vier Jahrzehnte Statistik, Forschung und Politikberatung. Festschrift für Karl Schwarz. Boppard am Rhein: Boldt, 169-186.

Lloyd, K.M. \& S.J. South. 1996. Contextual Influences on Young Men's Transition to First Marriage. Social Forces, 74 (3), 1097-1119.

Marini, M.M. 1984. Age and Sequencing Norms in the Transition to Adulthood. Social Forces, 63 (1), 229-244. 
Nauck, B. 1995. Regionale Millieus von Familien in Deutschland nach der politischen Vereinigung. In: B. Nauck \& C. Onnen-Isemann (Hrsg.), Familie im Brennpunkt von Wissenschaft und Forschung. Neuwied: Luchterhand, 91-121.

Nauck, B. \& H. Bertram (Hrsg). 1995. Kinder in Deutschland: Lebensverhältnisse von Kindern im Regionalvergleich. Opladen: Leske + Budrich.

Oppenheimer, V.K. 1988. A Theory of Marriage Timing. American Journal of Sociology, 94 (3), 563-591.

Oppenheimer, V.K. 1997. Women's Employment and the Gain to Marriage: The Specialization and Trading Model. Annual Review of Sociology, 23, 431-453.

Oppenheimer, V.K. 2000. The Continuing Importance of Men's Economic Position in Marriage Formation. In: L.J. Waite et al. (Hrsg.), The Ties That Bind. Perspectives on Marriage and Cohabitation. New York: Aldine de Gruyter, 283301.

Sackmann, R. \& H. Häussermann. 1994. Do regions matter? Regional differences in female labour-market participation. Environment and Planning A, 26 (6), 13771396.

Snijders, T.A.B. \& R.J. Bosker. 1999. Multilevel analysis: An introduction to basic and advanced multilevel modeling. London: Sage.

SOEP-Gruppe. 2001. The German Socio-Economic Panel (GSOEP) after more than 15 years - Overview. In: E. Holst et al. (Hrsg.), Proceedings of the 2000 Fourth International Conference of German Socio-Economic Panel Study Users (GSOEP2000), Vierteljahrshefte zur Wirtschaftsforschung, 70 (1), 7-14.

South, S.J. \& K.D. Crowder. 2000. The Declining Significance of Neighborhoods? Marital Transitions in Community Context. Social Forces, 78 (3), 1067-1099.

Teachman, J.D. \& K.D. Crowder. 2002. Multilevel Models in Family Research: Some Conceptual and Methodological Issues. Journal of Marriage and Family 64 (2): 280-294.

Waite, L.J., et al. (Hrsg.). 2000. The Ties That Bind. Perspectives on Marriage and Cohabitation. New York: Aldine de Gruyter. 


\section{Anhang}

Abbildung 1: Räumliche Verteilung durchschnittlicher roher Heiratsziffern in westdeutschen Stadt- und Landkreisen, 1986/93

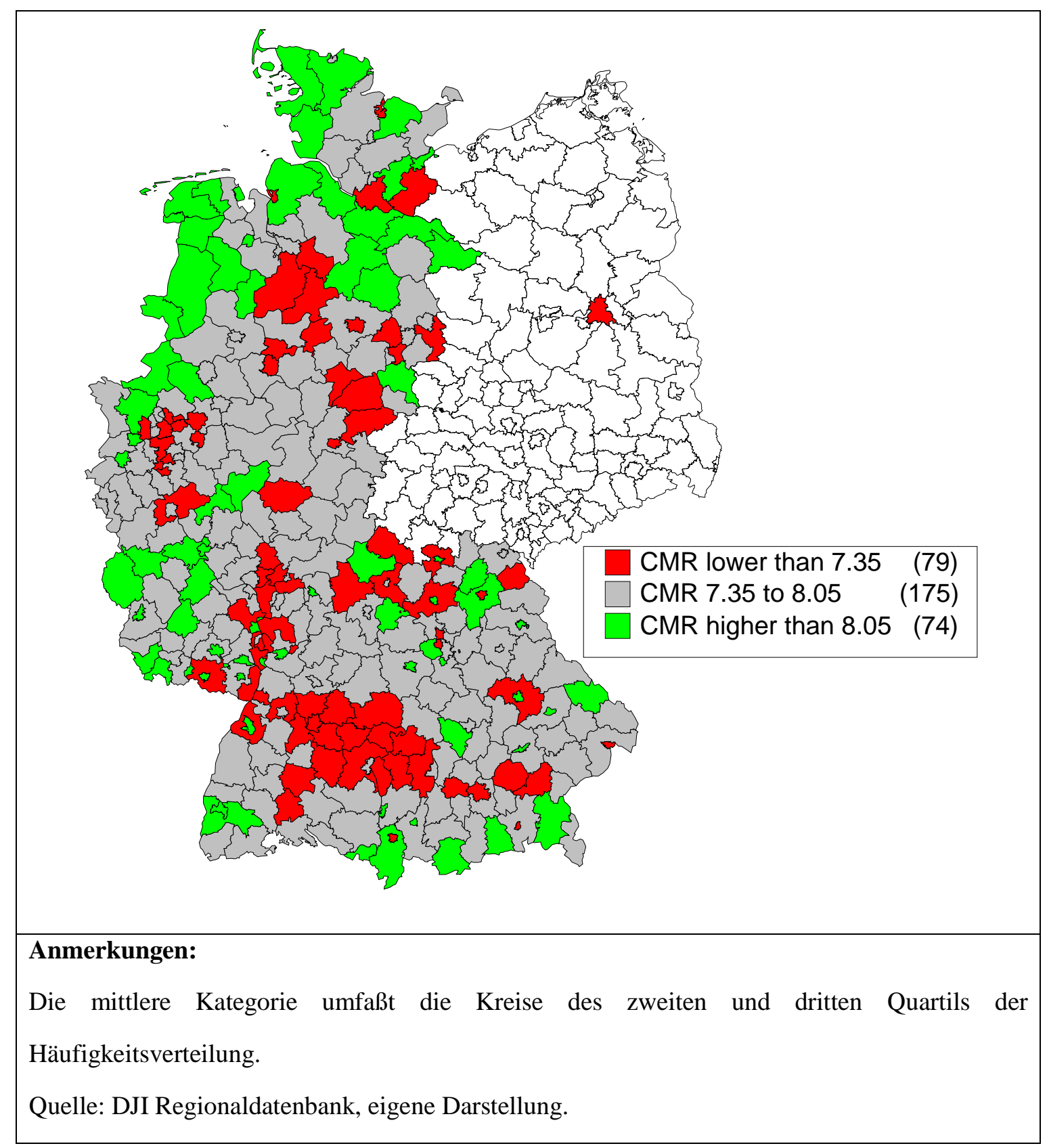


Tabelle 1: Deskriptive Statistiken

\begin{tabular}{|l|c|}
\hline Variablen & Mittelwert (Standardabweichung) \\
\hline Individualmerkmale & $26.0(4.8)$ \\
Alter & $698.4(268.3)$ \\
Quadriertes Alter & .20 \\
In Ausbildung & .18 \\
Kein Ausbildungsabschluss & .52 \\
Beruflicher Ausbildungsabschluss & .09 \\
Universitätsabschluss & .59 \\
Vollzeit erwerbstätig & .23 \\
Ausländer & \\
Kontextmerkmale & $48.6(0.8)$ \\
Frauen-Anteil & $41.2(3.4)$ \\
Frauenerwerbsquote & .17 \\
Ländlicher Raum & $8.6(3.1)$ \\
Arbeitslosenquote & 850 \\
\hline N (Ereignisse) & 300 \\
N (Kreise) & regional \\
N (Männer) & 2,880 \\
N (Beobachtungen) & 15,223 \\
\hline Anmerkungen: & \\
Mittelwerte beziehen sich auf beobachtete Personenjahre. & \\
Standardabweichungen für binäre Variablen werden nicht ausgewene & \\
Quelle: SOEP & Regionaldatenbank, \\
Berechnungen. & Statistik \\
\hline
\end{tabular}


Tabelle 2: Ergebnisse der zeitdiskreten logistischen Mehrebenenmodelle

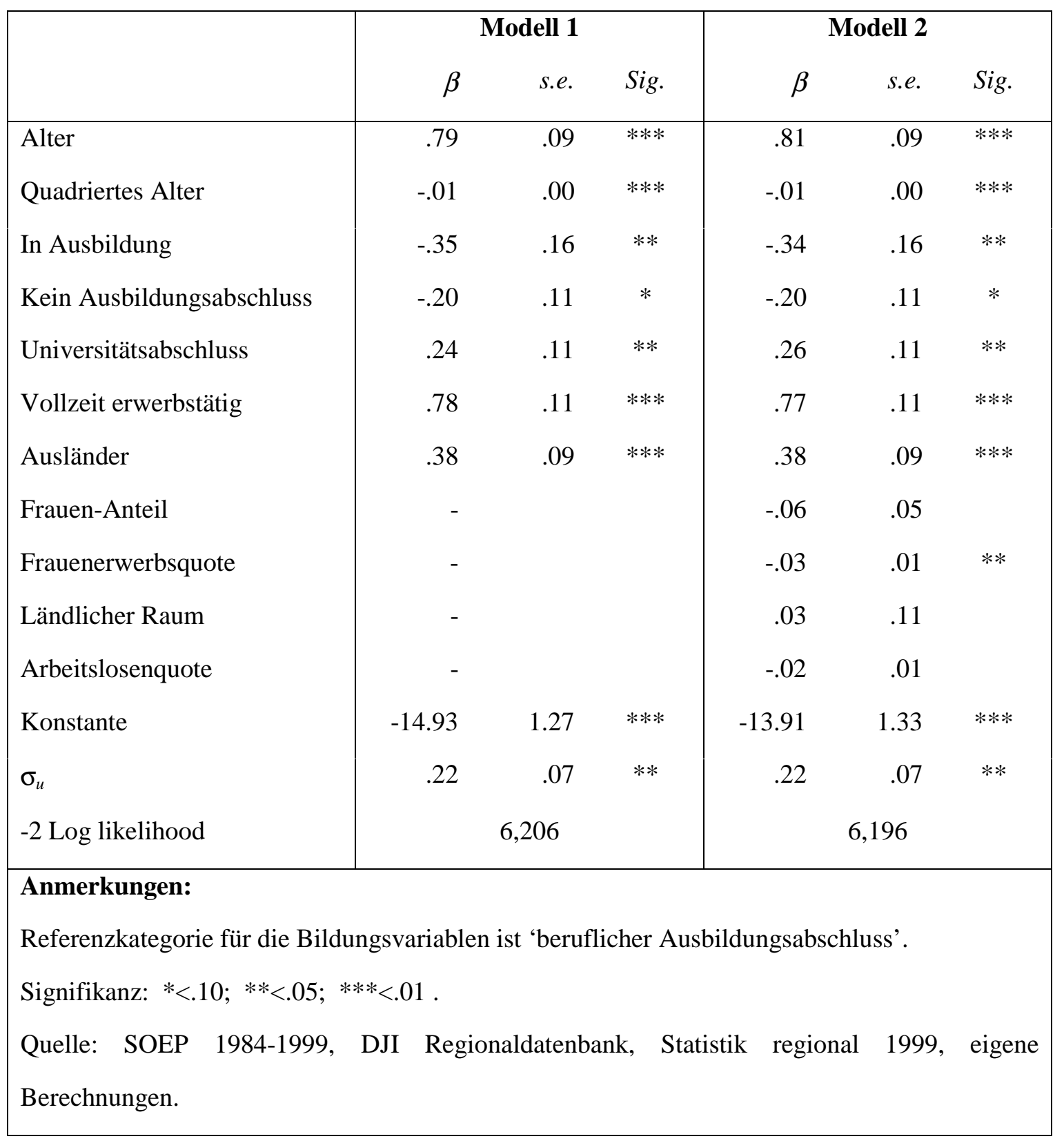

